\title{
DESIGN ISSUES FOR ELECTRONIC AUCTIONS
}

\author{
Jarrod Trevathan and Wayne Read \\ School of Mathematical and Physical Sciences \\ James Cook University \\ Hossein Ghodosi \\ School of Information Technology \\ James Cook University
}

Keywords: Auction security, anonymity, authentication, price determination, payment enforcement, group signatures.

Abstract: Extensive research has been conducted in order to improve the security and efficiency of electronic auctions. However, little attention has been paid to the design issues. This paper discusses design issues and contrasts the differing security requirements between various auction types. We demonstrate that poor design for an electronic auction breaches the security of the system and degrades its practicality, irrespective of how secure/efficient the building blocks of an electronic auction are. This is accomplished by illustrating design flaws in several existing electronic auction schemes. Furthermore, we provide a solution to these flaws using a group signature scheme and give recommendations for sound auction design.

\section{INTRODUCTION}

An auction is an exchange mechanism whereby a seller (or sellers) offers an item for sale and many bidders submit bids in competition for the item. The Auctioneer organises the auction on behalf of the seller and accepts bids from the bidders. The Auctioneer attempts to maximise the sale price for the seller, whereas the bidders try to win the item for the lowest price possible. An electronic auction is a cryptographic protocol designed to securely and anonymously conduct auctions. While extensive research has been undertaken for improving the security and efficiency of electronic auctions, (see (Cachin, 1999; Franklin and Reiter, 1996; Kikuchi et al., 1998; Naor et al., 1999; Trevathan, 2005)), little attention has been paid to the design issues. In this paper we demonstrate that poor design for an electronic auction breaches the security of the system and degrades its practicality regardless of how secure/efficient the building blocks of an electronic auction are. This is accomplished by illustrating design flaws in several existing auction schemes. We also provide solutions to these flaws using a group signature scheme and give some recommendations for sound auction design.

This paper is organised as follows: Section 2 gives a general background on electronic auction schemes. Section 3 describes the security problems inherent in conducting electronic auctions. Section 4 discusses design issues and illustrates some major design flaws in several existing schemes. Section 5 gives some recommendations about how these flaws can be fixed and suggestions regarding good auction design principles. Section 6 provides some concluding remarks.

\section{TYPES OF AUCTIONS}

There are many types of auctions, including English, Vickrey, Dutch, and Continuous Double auctions (see Figure 2). An English auction is the most prominently known type of auction and is commonly used in real estate. In this auction there is one seller who offers an item for sale. Many potential buyers submit bids in an attempt to win the item. The winner is the highest bidder after a given time-out period.

An alternative to this is the Vickrey auction. This is referred to as a sealed bid, second price auction. It is comprised of bidders submitting their bids secretly during a bid submission round. The bids remain sealed until a winner determination round, where the Auctioneer views all of the bids and awards the auction to the highest bidder. In this auction, the winner only has to pay the second highest price, i.e., the highest losing bid. The values of all other losing bids remain secret. In contrast, an English auction is an open bid, first price auction. 


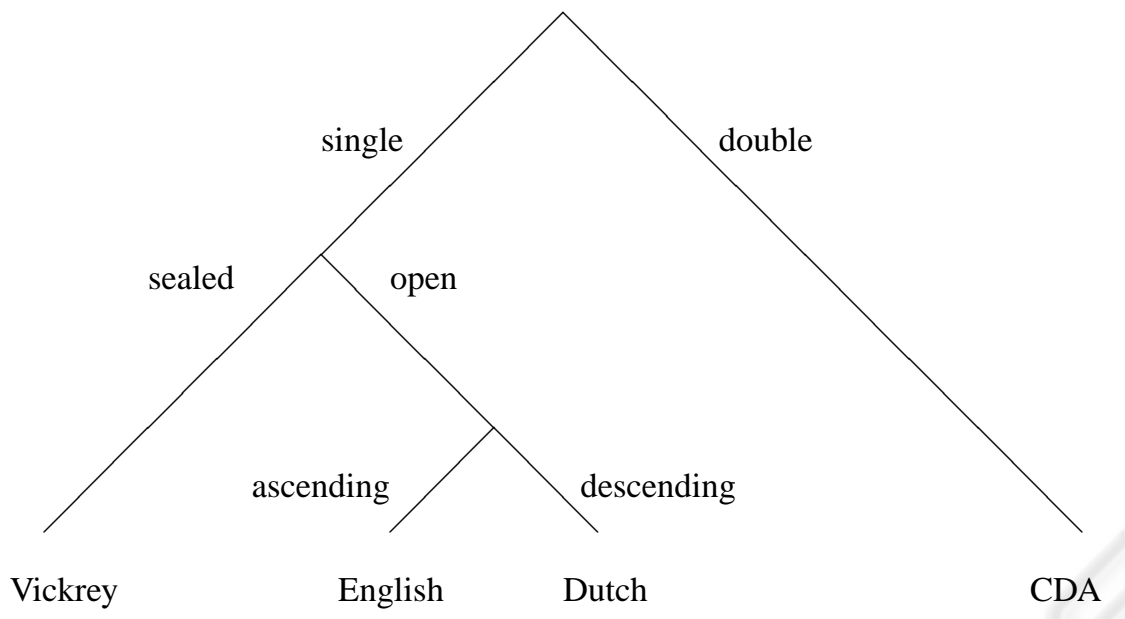

Figure 1: Taxonomy of Electronic Auction Schemes

In a Dutch or descending auction, the seller progressively lowers his/her bid until a buyer accepts. The winner is the first buyer to accept the seller's offer. They must pay an amount equal to this bid. In this respect Dutch auctions have the natural property of concealing the losing bid values. This form of auctioning is also referred to as descending, as unlike an English auction, the winning price is bid downwards rather than up.

A further style of auction used in share markets is a Continuous Double Auction (CDA). The aforementioned auctions have only one seller and many buyers. CDAs on the other hand, have many buyers and many sellers who continuously trade a particular commodity. These auctions are open bid and the price can be either ascending or descending.

Electronic auction schemes have been proposed for all of these auction types. However, regardless of the auctioning mechanism, all schemes consist of the following stages:

Initialisation: The Auctioneer sets up the auction and advertises it (i.e., description of good, starting time, etc).

Registration: In order to participate in the auction, bidders must first register with the Auctioneer (or a registration manager). This ensures only valid bids are made and bidders can be identified for payment purposes. It is desirable for registration to be a oneoff procedure. When a bidder has registered they are able to participate in any number of auctions rather than re-registering for each new auction.

Bidding: A registered bidder computes his/her bid and submits it to the Auctioneer. The Auctioneer checks the bid received to ensure conformity with the auction rules.

Winner Determination: The Auctioneer determines the winner according to the auction rules. It is desir- able for this process to be publicly verifiable.

\section{AUCTION SECURITY}

In recent years, many companies have emerged offering auctioning services via the Internet (e.g., eBay ${ }^{1}$ and onSale ${ }^{2}$ ). Such sites lack security for both the seller and the bidder and moreover require all parties to trust the Auctioneer. Furthermore, the identities and information relating to the participants is open to abuse. Common problems identified include: the bidders repudiating bids (i.e., they win and later decide they don't want to pay), the seller not delivering the item and Auctioneer corruption by awarding the auction to someone other then the legitimate winner.

Security and anonymity has been addressed in literature by (Cachin, 1999; Franklin and Reiter, 1996; Kikuchi et al., 1998; Naor et al., 1999; Trevathan, 2005). Additionally, numerous auction schemes have been proposed in an attempt to solve the aforementioned problems. There are differing security requirements depending on whether the auction is sealed or open bid. However, in general, most schemes in literature agree on the following security goals:

Unforgeability - Bid must be unforgeable, otherwise a bidder can be impersonated.

Non-Repudiation - Once a bidder has submitted a bid they must not be able to repudiate having made it. For example, if a bidder wins and does not want to pay they might deny that they submitted the bid.

Anonymity - The bidder-bid relationship must be

\footnotetext{
${ }^{1} \mathrm{http}: / / \mathrm{www} . e b a y . c o m$

${ }^{2} \mathrm{http}: / /$ www.onsale.com
} 
concealed so that no bidder can be associated with the bid they submit.

Public Verifiability - There must be publicly available information by which all parties can be verified as having correctly followed the auction protocol. This should include evidence of registration, bidding and proof of winner/loser.

Robustness - The auction process must not be affected by invalid bids nor by participants not following the auction protocol correctly.

\section{DESIGN ISSUES}

The main design goals discussed previously in the literature of electronic auctions include fairness, efficient registration, efficient bidding, and efficient winner determination (see, for example, (Boyd and Mao, 2000; Franklin and Reiter, 1996; Naor et al., 1999; Boyd et al., 2000; Trevathan, 2005)). Our observation is that despite all components of an electronic auction functioning correctly, there is still no guarantee that the whole system works properly. In this section we discuss several design issues that must be considered in order to achieve a practical auction system.

\subsection{Trust Issues}

Auctioneer corruption is a major problem in electronic auctions. A malicious Auctioneer might influence the auction proceedings in a manner inconsistent with the auction rules. For example, the Auctioneer might choose to block bids, insert fake bids, steal payments, profile bidders, open sealed bids prior to the winner determination phase, or award the item to someone other than the legitimate winner. Furthermore, the Auctioneer may be in collusion with some of the bidders.

In general, all schemes can be classified according to how they deal with this problem. We have identified the following trust models:

Auctioneer Trust - This is the easiest (and the most unacceptable) solution to the problem. It is assumed that the Auctioneer is trustworthy and honestly follows the protocol. This is the strategy employed by all Internet auction sites as it easily solves many relevant problems (e.g., security, anonymity, etc.). However, all bidders are at the mercy of the company running the auction.

Trusted Third Party - Since the Auctioneer is a beneficiary, the assumption that it follows the auction protocol is unrealistic. An alternative could be that the bidders and Auctioneer provide a trusted third party (TTP) with information so that when there is a dispute the TTP can be called upon to resolve it (see (Boyd et al., 2000)). However, such a setup requires all parties to have confidence in the TTP who is an attractive security target and a bottleneck.

Threshold Trust - Threshold trust schemes protect against a corrupt Auctioneer by distributing the role of the Auctioneer across $\ell$ servers (see (Franklin and Reiter, 1996; Sakurai and Miyazaki, 1999)). The auction can be considered secure/fair unless a threshold $t, 1 \leq t \leq \ell$ of the Auctioneers collude. The value of $t$ is usually around $\ell / 3$.

Such a scheme is clearly better then the previous approaches as no single party acting maliciously can influence the auction proceedings. However, threshold trust requires much communication between bidders and the auction servers, as well as between the auction servers themselves. Furthermore, when the auctioning company is small, collusion among Auctioneers is beneficial to the group as a whole.

Two-Server Trust - An alternative approach to threshold trust is to split the auctioning responsibility among two servers owned by separate entities (see (Cachin, 1999; Naor et al., 1999)). Here the auction result can be trusted as long as the two entities do not collude. Two-server trust schemes effectively reduce the communication overhead involved in threshold trust schemes and thus far have proven to be computationally efficient. However, if one of the two servers decides not to co-operate, then the auction outcome cannot be determined.

Distributed Trust - In this approach the bidders jointly calculate the auction result without the help of an Auctioneer. The merit of such an approach is that collusion amongst bidders is prevented unless all bidders are corrupt, which negates the reason for colluding in the first place (see (Brandt, 2003)).

Distributed trust schemes are unrealistic as they require all bidders to participate during the winner determination phase. Such an approach is not feasible when the number of bidders is large. Furthermore, distributed trust is not applicable to CDAs as this assumes knowledge of the total number of bidders prior to the start of bidding. In a CDA, new bidders are entering the auction all the time. Using a distributed bidder approach would require communicating new information to all the auction participants every time a new bidder registers.

\subsection{Anonymity Issues}

Another important criteria for the evaluation of secure electronic protocols is anonymity. This is important because the bidder-bid relationship must be concealed in such a way that no bidder can be associated with the 
bid they have submitted. There are several different approaches to satisfy this requirement. For example, auction protocols which utilise secure computation for winner determination (e.g., (Kikuchi et al., 1998; Harkavy et al., 1998) provide this facility by concealing the identity of bidders in their bids). A common solution is to issue bidders with a pseudonym (during registration), which they can use to submit bids. That is, bidders register themselves (by presenting verified identification) and obtain a pseudonym.

Seemingly the technique of issuing pseudonyms requires having more than one server/party on the auction side. Otherwise, the same party issuing the pseudonym knows the real identity of the associated bidder, and thus learns the relationship between the bids and the bidders. On the contrary, if the pseudonym issuer does not know the pseudonym (e.g., it is issued by blindly signing a message), then it cannot retrieve the real identity of a bidder in the case of a dispute. This model essentially works as follows (assume the two servers/parties are called $S_{1}$ and $S_{2}$ ):

1. Bidders present their identification to $S_{1}$ and obtain a token that does not carry their ID.

2. Bidders submit the token (without revealing their ID) to $S_{2}$, who issues a pseudonym associated with the token.

In this way, neither $S_{1}$ nor $S_{2}$ knows the relationship between any real ID and the pseudonyms. The bidder then submits his/her bid using the pseudonym. However, in the event of a dispute $S_{1}$ and $S_{2}$ can cooperate to determine the real ID associated with each pseudonym.

Our observation is that, regardless of how secure the anonymity issuing protocol is, the resulting scheme is not secure if there is no separation between the registration and the bidding phases. That is, the registration must be performed for all bidders prior to the commencement of bidding and the system must not accept any bid before the registration is closed. The following scenario explains a possible attack: A bidder provides identification to $S_{1}$ and obtains a token. Using this token, it obtains a pseudonym which can be used for bidding. If there is no separation between the registration and bidding phases $S_{1}$ can act in a procrastinating manner by halting all future registrations until the newly registered bidder submits his/her bid. This scenario enables $S_{1}$ to learn the mapping between the bidder's identity and his/her pseudonym. To protect against this type of attack, the scheme should not allow any bidding prior to the registration closing time.

Note that there are electronic auction schemes in which it is impossible to have a separation between the registration and the bidding phases. For example, a CDA allows bidders to continuously submit bids at the same time new bidders are being registered (i.e., the registration and bidding phases overlap). Therefore the scheme by Wang and Leung (Wang and Leung, 2004) can be broken using this procrastinating attack.

\subsection{Bid Authentication Issues}

Efficient winner determination is an important criteria in the evaluation of electronic auction protocols. Because of its importance, many schemes provide evidence to support their claims regarding the ability of their system to efficiently process bids. For example, (Franklin and Reiter, 1996) in their highly referenced work, claim that:

"We have implemented a prototype of our service to demonstrate its feasibility. The performance of this implementation indicates that our approach is feasible using off-the-shelf workstations for auction servers, even for large auctions involving hundreds of bids."

The question is, what will happen if a (set of) malicious bidder(s) issues too many bids? Optimistically assuming that processing each bid takes only one second, then the winner determination process will require a proportional amount of time (i.e., the system is not practical). The problem is more crucial in schemes which use secure computation (see, e.g., (Kikuchi et al., 1998)). They achieve anonymity by concealing the identity of bidders in their bids. In addition, all bids are submitted anonymously. The winner determination protocol opens only one bid -a bid that contains the highest offer. If there is a tie (i.e., more than one bid at the highest offer), then another round of bidding must occur. A malicious bidder can easily cause a never ending scenario in this scheme. For example, even if all high valued bids are opened (which is disallowed by the protocol), the Auctioneer cannot determine who has submitted the bid as it contains a false identity. Obviously, such schemes are not practical at all.

This problem can be avoided if the bids have been authenticated. That is, the Auctioneer accepts bids only from registered bidders (generally, in all sealed-bid auctions, each bidder submits only one bid). If the system supports anonymity of the bidders, then it must also provide the authentication of the corresponding pseudonym. Note that there are schemes which check the validity of the bids (i.e., they check whether the submitted bid satisfies a predetermined structure). This is insufficient, as one may submit too many well-structured bids. Furthermore, bid authentication must be secure about relevant attacks. In order to illustrate the problem, let us examine the protocol by (Boyd et al., 2000), which supports a sealed bid auction system. This is possibly the only auction scheme which uses bid 
Table 1: The Sealing Protocol

\begin{tabular}{|ccc|}
\hline Bidder & Auctioneer \\
\hline$a, d_{1}, d_{2} \in_{R} Z_{q}^{*}$ & $\stackrel{S, B}{\longrightarrow}$ & \\
$S=g_{1}^{b} g_{2}^{a}, B=g_{1}^{d_{1}} g_{2}^{d_{2}}$ & $\stackrel{\leftrightarrow}{c}$ & $c \in_{R} Z_{q}$ \\
$s_{1}=d_{1}-c x_{1}, s_{2}=d_{2}-c x_{2}$ & & \\
$t_{1}=s_{1}-b c, t_{2}=s_{2}-a c$ & $\stackrel{t_{1}, t_{2}}{\longrightarrow}$ & $\stackrel{?}{=}\left(S y_{1} y_{2}\right)^{c} g_{1}^{t_{1}} g_{2}^{t_{2}}$ \\
\hline
\end{tabular}

authentication. But, as we will show shortly, it is subject to an impersonation attack.

System Settings in (Boyd et al., 2000) -

$G$ is a subgroup of order $q$ in $Z_{p}^{*}$, such that $p=2 q+1$ for sufficiently large prime $p$. There are two generators, $g_{1}$, and $g_{2}$, such that nobody knows $\log _{g_{1}} g_{2}$. The public keys of a bidder $b_{i}$ are certified to be $y_{1}=g_{1}^{x_{1}}$ and $y_{2}=g_{2}^{x_{2}}$.

Sealing Protocol in (Boyd et al., 2000) -

This is an interactive protocol between the bidder and the Auctioneer. At the end of this protocol, the Auctioneer will be convinced that the bidder submitted a correct bid which can be opened at the bid-opening phase (in their scheme, the help of the bidder is necessary to open the bid). A bidder, $b_{1}$, with public keys $y_{1}=g_{1}^{x_{1}}$ and $y_{2}=g_{2}^{x_{2}}$ wishes to commit himself/herself to the bid value $b$. The protocol has three steps and works as shown in Table 1.

To open the bid (at the bid-opening phase) the bidder should release the tuples $(b, a)$. This tuple can be checked with corresponding values at the bid sealing protocol, and upon verification, the value $b$ is the bid submitted by bidder $b_{1}$. If a bidder refrains from opening his/her bid (e.g., when he/she learns that his/her bid is too much higher than the others), the identity of this person can be traced and suitable action will be taken. The idea behind this protocol is that the tuple $\left(S, B, c, t_{1}, t_{2}\right)$ is unique, and thus the bidder is committed to the bid value $b$. The authors also proposed a non-interactive version of this protocol (see (Boyd et al., 2000)).

Anyone that does not know the secret keys $x_{1}$ and $x_{2}$, cannot participate in, and complete the protocol successfully. The impersonation attack works as shown in Table 2 (the non-interactive version is also subject to this attack).

The consequence of this attack is that an innocent bidder $b_{1}$ will be accused of not participating in the bid opening protocol. But, in fact, nobody knows the relevant tuple $(b, a)$ for this case (if solving the Dis-
Table 2: The Sealing Protocol by a Malicious Bidder

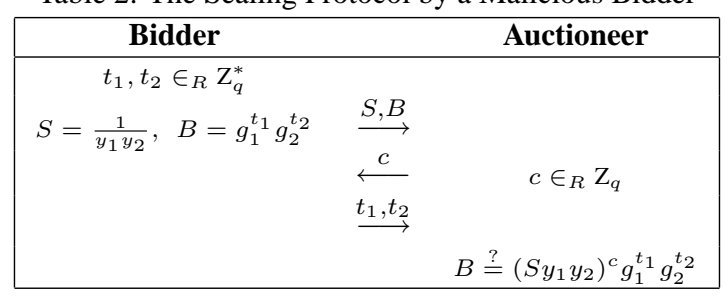

crete Logarithm problem is hard).

\subsection{Price Determination Issues}

Many auction schemes impose a price list on bidders (e.g., (Harkavy et al., 1998; Sakurai and Miyazaki, 1999)). This is essentially a series of bidding points $\left(w_{1}, w_{2}, \ldots, w_{k}\right)$ where $w_{1}<w_{2}<\ldots<w_{k}$ which a bidder must choose from.

The scheme by (Harkavy et al., 1998) requires bidders to submit a bid value for each point in the price list. Meaning, for each point in the price list, a bidder must either bid his/her $i d$ or a nullifying value. For a price list of size $k$, this requires a bidder to submit $k$ individual values. In some types of auctions (such as CDAs), a price list may be too restrictive as bidders require flexibility in choosing their bid. In some instances this may be down to fractions of cents. Adding more bidding points comes at the cost of increased computation and communication overhead. So the question here is what is the correct value of $k$ to choose?

Moreover, two colluding bidders can continually bid at the highest price level and cause infinite rounds of auctioning (i.e., denial of service attack). This is a major problem with the schemes of (Harkavy et al., 1998) and (Sakurai and Miyazaki, 1999). For example, if the highest price in the list is $k$ and two bidders bid at this price, then the auction goes to a second round of bidding. In this round the maximum value in the price list increases to $k \times 2$. However, both bidders then bid $k \times 2$. This then forces a third round of bidding and the maximum value in the price list increases to $k \times 3$, etc. If the bidders continue in this manner, the auction continues indefinitely.

In addition, many schemes using a price list, also require bidders to participate in a disavowal protocol (e.g., (Sakurai and Miyazaki, 1999)). This requires the bidder to engage in a protocol with the Auctioneer to show that the bid they submitted is not for that particular price. Upon completion of disavowal for the respective price level, the Auctioneer selects the next price in the list and the process repeats. Unfortunately disavowal protocols are very expensive as much communication must take place to determine the winner. 
Furthermore, if some bidders do not participate they are instantly incriminated, or the auction process is left in an inconsistent state.

For the reasons stated above, schemes using price lists tend to be restrictive and are not always practical for certain types of auctions.

\subsection{Payment Issues}

A major issue in electronic auction schemes is how to enforce payment from the winning bidder(s). Previous schemes have achieved this by using digital cash schemes. For example, Franklin and Reiter (Franklin and Reiter, 1996) use a digital coin to represent a bidder's bid. The value of the coin is equivalent to the amount of the bid. The winner's coin is deposited into the seller's bank account at the end of the protocol. In this scheme the purpose of the coin is to prevent repudiation of bids. The scheme by (Boyd et al., 2000), uses a digital cash scheme in a similar manner. When a bidder repudiates a bid, the identity of the bidder can be recovered by presenting the bidder's piece of digital cash to the bank.

We believe that using digital cash does not significantly enhance the security of an electronic auction scheme. Instead it introduces another party (i.e., the bank) to the auction which must be trusted by the participants. This complicates auction schemes and makes it increasingly difficult to analyse how secure the scheme is. Franklin and Reiter (Franklin and Reiter, 1996) claim that their scheme can provide anonymity through the use of pseudonyms. However even if this is done, anonymity is still dependent on the underlying digital cash scheme.

The main goal of digital cash is to allow a spender to engage in anonymous and untraceable transactions with a merchant. When the spender breaks the rules (i.e., repudiates the purchase or double spends the cash) the only means of recourse is for the bank to reveal the spender's identity. In terms of an anonymous auction, we are only interested in revealing a bidder's identity when there is a dispute (i.e., bid repudiation). Unless the bank is built into the auctioning model (as in (Boyd et al., 2000)), other more efficient mechanisms for identity escrow can be used rather than digital cash schemes.

\section{RECOMMENDATIONS}

This section provides some suggestions of ways to fix the problems raised in the previous section and discusses good design strategies for electronic auctions. Surprisingly there already exists cryptographic mechanisms to solve many of the problems facing electronic auctions. However, little if any of the literature on electronic auctions has embraced this research. The proposed solution comes from group signature schemes.

A group signature scheme allows members of a group to sign messages on behalf of the group so that no one can work out which particular user is the signer (see (Anteniese et al., 2000)). A signature on a message can be verified by anyone using a single public key. When there is a dispute (i.e., someone repudiates having signed a message) a trusted group manager can reveal the identity of the signer of a message. Each signature is unlinkable and no coalition of users (even with the help of the group manager) is able to forge a valid signature of an innocent user.

Group signatures have many desirable properties with regard to electronic auctions. In an auction, each bidder belongs to a group of bidders. Every bidder has a unique signature which enables them to sign bids anonymously. The bidders submit their bids to the Auctioneer, who can then verify each signature using the group's public key. However, the Auctioneer cannot solely be the group manager as this would require all bidders to implicitly trust that the Auctioneer would not reveal their identities. Instead this task must be distributed among the Auctioneer and another party (e.g., a registration manager). When there is a dispute, both the Auctioneer and the other party must cooperate in order to reveal the identity of whom signed the bid in question.

In the subsections that follow, we will address each of the design issues stated in the previous section and describe how the properties of group signature schemes can be employed. Throughout this section, we develop a model for electronic auction design. This model is not specific to any particular auction type, but can be used as a general basis for all types of auctions. Note that mechanisms for sealing bids will not be discussed. Instead the model addresses trust, anonymity, bid authentication, price flexibility and payment enforcement.

\subsection{Trust}

Using publicly verifiable protocols reduces the trust required in the Auctioneer. This approach allows all parties (even outsiders) to view the auction process and they can verify that the auction outcome is correct.

Many schemes use public bulletin boards to serve this purpose (Sakurai and Miyazaki, 1999; Boyd et al., 2000; Wang and Leung, 2004). An Auctioneer can post bids and auction results on the board so that all others can verify the auction proceedings. It is assumed that only the Auctioneer can write to this board and that it is publicly accessible to all parties. 
Public bulletin boards have implications for sealed bid auctions as the main requirement is to keep the losing bids secret. However, in all sealed bid auction schemes it is inevitable that bidders learn statistics about the bids submitted (i.e., highest price, bid values, etc). Therefore, using a bulletin board is sufficient for sealed bid auctions, as long as the bidderbid relationship remains secret (i.e., bidders cannot be linked to their bids).

However, even if publicly verifiable protocols are used there is still some need to trust the Auctioneer. In this case, the best approach is to use the two-server trust model (see Section 4.1). This needs to be modified slightly in that the second party is only required in the case of a dispute but cannot act on his/her own (as in the case of the TTP approach). This will be clarified in the next section.

\subsection{Anonymity}

To protect against the procrastinating attack described in section 4.2 , there must be complete separation between the registration and bidding stages of an auction. This means that all bidders must register prior to the Auctioneer accepting any bids. This is a straightforward matter for English, Vickrey and Dutch auctions as the Auctioneer can prevent the auction from starting until all bidders have registered.

However, in CDAs it is impossible to separate the registration and bidding stages. In this situation a group signature scheme can be used. Group signatures are unlinkable, which means that given two signatures, it is impossible to tell if the same user signed both messages. This thwarts the procrastinating attack as $S_{1}$ can no longer determine if the signature that they are observing belongs to the newly registered bidder or someone else. Even if $S_{1}$ does learn the mapping between the newly registered bidder and the signature on a bid, the registration manager will not be able to link future bids to the bidder due to the unlinkability property of the group signature.

\subsection{Bid Authentication}

In general, authentication protocols require considerable time consuming operations such as exponentiation. However, there are algorithms that can be employed for performing multiple verifications in one stage/operation (e.g., (Bellare et al., 1998)). Group signatures are also an effective method of performing bid authentication.

\subsection{Price Determination}

Bidders must be allowed flexibility when choosing their bid values. Using a group signature approach fa- cilitates price flexibility as bidders are free to choose whatever bid values they desire. Furthermore, a group signature scheme does not require the use of a disavowal protocol. In general, designers of electronic auctions should avoid disavowal protocols.

\subsection{Payment Enforcement}

As stated in Section 4.5, digital cash schemes only serve as a non-repudiation mechanism in auctions and makes the scheme conceptually harder to understand. There are more efficient identity escrow mechanisms other then digital cash. Again group signature schemes are an ideal choice. When there is a dispute, (i.e., someone repudiates a bid, or refuses to pay), the group manager can reveal the identity of the person that signed the bid in question. This is more efficient then a digital cash scheme and serves the same purpose.

\subsection{Group Signature Auction Model}

This section proposes a generic auction model using a group signature scheme. The model incorporates the recommendations from the previous subsections. Furthermore, it gives the designer flexibility to use any group signature scheme.

There are three parties in the auction:

1. A Bidder, who is interested in submitting bids for an item offered by a seller.

2. An Auctioneer, who organises the auction, accepts the bids and determines the winner according to the auction rules. The Auctioneer also holds the corresponding relation of the identity and a token associated with each bidder. The Auctioneer has access to a publicly verifiable bulletin board.

3. A Registrar, who takes part in a protocol in order to complete the registration of a bidder who has obtained a token from the Auctioneer. At the end of the protocol, the bidder obtains a secret key that enables him/her to generate signed bids in a proper format.

Setup - The Auctioneer organises the auction (i.e., advertising and calls for auction). The Registrar sets up the group public key and his secret key.

Registration - A user submits a request to the Auctioneer to participate in the auction. The Auctioneer verifies the identity of the requestor, and issues a token that is verifiable by the Registrar. The user then takes part in a protocol with the Registrar, in order to obtain his secret key and a certificate of membership in the auction. Note that the token does not carry the real identity of the bidder, but all communication between the Registrar and the owner of a token is authenticated, and will be kept for tracing, or revealing the identity of users associated with tokens. 
Bidding - Using a membership certificate, a bidder can generate anonymous and unlinkable group signatures on a bid. A bidder submits a bid to the Auctioneer signed using the secret key. The Auctioneer verifies the signature on the bid using the public key. If the bid is valid, the Auctioneer posts it on the bulletin board.

Winner Determination - The Auctioneer determines the auction outcome according to auction rules (i.e., English, Vickrey, etc.). The auction result is posted on the bulletin and the winner can produce his/her signed bid as evidence that they won.

Traceability - In the event of a dispute (i.e., bid repudiation), the Auctioneer and the Registrar can combine their information to reveal the identity of a signer.

\section{CONCLUSION}

Limited attention has been paid to the design issues of electronic auctioning schemes. This paper demonstrated that poor auction designs breach the security of any auction scheme irrespective of how secure the underlying cryptographic building blocks are. Auctions were examined in terms of trust, anonymity, bid authentication, price determination and payment enforcement.

We have shown that many schemes proposed in literature are not practical and can be broken by exploiting weakness in the fundamental design employed. A group signature scheme can solve many of the problems in electronic auction design. However, this is not an all-encompassing solution to all of the design issues. It is insufficient to rely on cryptographic mechanisms alone. An auction system is only secure as its weakest component.

There still remain many pressing issues in designing electronic auctions. Now the time has come for the designers of auction schemes to step away from the small-view approach and observe the auction system as a whole. Designers need to analyse how each component interacts and scrutinise whether the cryptographic methods employed are really sufficient for use in electronic auction schemes.

\section{REFERENCES}

G. Ateniese, J. Camenisch, M. Joye, and G. Tsudik, "A Practical and Provably Secure Coalition-Resistant Group Siganture Scheme," in Advances in Cryptology - Proceedings of CRYPTO 2000 (M. Bellare, ed.), vol. 1880 of Lecture Notes in Computer Science, pp. 255-270, Springer-Verlag, 2000.
M. Bellare, J. Garay, and T. Rabin, "Fast Batch Verification for Modular Exponentiation and Digital Signatures," in Advances in Cryptology - Proceedings of EUROCRYPT '98 (K. Nyberg, ed.), vol. 1403 of Lecture Notes in Computer Science, pp. 236-250, SpringerVerlag, 1998.

C. Boyd and W. Mao, "Security Issues for Eelctronic Auctions," tech. rep., Hewlett Packard, TR-HPL-2000, 2000.

F. Brandt, "Fully private auctions in a constant number of rounds," in Proceedings of the 7th Annual Conference on Financial Cryptography (FC) (R. Wright, ed.), vol. 2742 of Lecture Notes in Computer Science, pp. 223238. Springer-Verlag, 2003.

C. Cachin, "Efficient Private Bidding and Auctions with an Oblivious Third Party," in 6th ACM Conference on Computer and Communication Security, pp. 120-127, 1999.

M. Franklin and M. Reiter, "The Design and Implementation of a Secure Auction Service," IEEE Transactions on Software Engineering, vol. 22, pp. 302-312, May 1996.

M. Harkavy, J. Tygar, and H. Kikuchi, "Electronic Auctions with Private Bids," in the 3rd USENIX Workshop on Electronic Commerce, Aug. 1998.

H. Kikuchi, M. Harkavy, J. Tygar, "Multi-round Anonymous Auction Protocols," IEEE Workshop on Dependable and Real-Time E-Commerce Systems, pp. 62-69, 1998.

M. Naor, B. Pinkas, and R. Sumner, "Privacy Preserving Auctions and Mechanism Design," in The 1st Conference on Electronic Commerce, pp. 129-139, 1999.

K. Sakurai and S. Miyazaki, "A Bulletin-Board Based Digital Auction Scheme with Bidding Down Strategy," in International Workshop on Cryptographic Techniques and E-Commerce, pages 180-187, 1999.

J. Trevathan, "Security, Anonymity and Trust in Electronic Auctions," Association for Computing Machinery, Crossroads Magazine, Spring Edition, vol. 11.3, 2005.

K. Viswanathan, C. Boyd, and E. Dawson, "A Three Phased Schema for Sealed Bid Auction System Design," in Proceedings of ACISP 2000 -Australasian Conference on Information Security and Privacy (E. Dawson, A.Clark, and C. Boyd, eds.), vol. 1841 of Lecture Notes in Computer Science, pp. 412-426, SpringerVerlag (Berlin), 2000.

C. Wang and H. Leung, "Anonymity and Security in Continuous Double Auctions for Internet Retails Market," in the $37^{\text {th }}$ Hawaii International Conference on Systems Sciences, 2004. 